\title{
Manuscript
}

Click here to view linked References

\section{Numerical simulation of pressure-driven displacement of a viscoplastic material by a Newtonian fluid using the lattice Boltzmann method}

\author{
Pinakinarayan A. P. Swain, George Karapetsas ${ }^{\dagger}$, Omar K. Matar ${ }^{\dagger \dagger}$ and \\ Kirti Chandra Sahu ${ }^{1}$ \\ Department of Chemical Engineering, Indian Institute of Technology Hyderabad, \\ Yeddumailaram 502 205, Andhra Pradesh, India \\ ${ }^{\dagger}$ Department of Mechanical Engineering, University of Thessaly, Greece \\ ${ }^{\dagger \dagger}$ Department of Chemical Engineering, Imperial College London, South Kensington \\ Campus, SW7 $2 A Z$.
}

\begin{abstract}
The pressure-driven displacement of a non-Newtonian fluid by a Newtonian fluid in a two-dimensional channel is investigated via a multiphase lattice Boltzmann method using a non-ideal gas equation of state well-suited for two incompressible fluids. The code has been validated by comparing the results obtained using different regularized models, proposed in the literature, to model the viscoplasticity of the displaced material. Then, the effects of the Bingham number, which characterises the behaviour of the yield-stress of the fluid and the flow index, which reflects the shear-thinning/thickening tendency of the fluid, are studied. It was found that increasing the Bingham number and increasing the flow index increases the size of the unyielded region of the fluid in the downstream portion of the
\end{abstract}

\footnotetext{
${ }^{1}$ Email: ksahu@iith.ac.in, Tel: +91 402301 6053, Fax: +91 4023016032 
channel and increases the thickness of the residual layer of the fluid resident initially in the channel; the latter is left behind on the channel walls by the propagating 'finger' of the displacing fluid. This, in turn, reduces the growth rate of interfacial instabilities and the speed of finger propagation. Keywords: Multiphase flow, Non-Newtonian Fluids, Lattice Boltzmann Simulation, Immiscible Fluids, Instability, Laminar Flow.

\section{Introduction}

Pressure-driven displacement flows of one fluid by another having different fluid properties are common in many industrial processes, such as enhanced oil recovery [1], the transportation of crude oil in pipelines [2], fixed bed regeneration, hydrology and filtration. In food processing industries, cleaning also involves the removal of highly viscous material from conduits via displacement by water streams. In flow through porous media or in Hele-Shaw cells, the displacement of a highly viscous fluid by a less viscous one is accompanied by viscous fingering [3]. Achieving fundamental understanding of these flows became an active research area for decades [4].

The dynamics of displacement flows have been investigated both numerically and experimentally by several authors by considering miscible [5-12] as well as immiscible fluids [13-18]. It is well known that the displacement flow is always stable when the invading fluid is more viscous than the resident fluid [2]. When the displacing fluid is less viscous, the flow becomes unstable and "roll-up" (in miscible flows $[1,19]$ ) and sawtooth 
structures (in immiscible flows, [18]) appear at the interface separating the fluids. The linear instability in the three-layer/core-annular flow, which can be obtained when the elongated "finger" of the less viscous fluid penetrates into the bulk of the more viscous one, was also studied in immiscible [20-22] and miscible [19, 23-26] systems.

In a Hele-Shaw cell, Goyal and Meiburg [7] studied numerically the miscible displacement flow of a highly viscosity fluid by a less viscous one. They observed that the two-dimensional instability patterns become three-dimensional at higher flow rates. The flow field obtained in their simulation was qualitatively similar to that observed in the experiment of Petitjeans and Maxworthy [8] and the theoretical predictions of Lajeunesse et al. [27]. In the context of enhanced-oil recovery, Taghavi et al. [10, 11] studied analytically and experimentally the displacement flow of two miscible fluids and observed Kelvin-Helmholtz like instabilities at low imposed velocities in the exchange flow dominated regime. Sahu et al. [9] investigated the effects of Reynolds number, Schmidt number, Froude number and angle of inclination in the pressure-driven flow of two miscible liquids of different densities and viscosities in an inclined channel. The behaviour of an infinitesimally small disturbance in such flows was also investigated by Sahu et al. [19] via a linear stability analysis.

The work discussed above considered only Newtonian fluids. In literature, to the best of our knowledge, very few studies has been carried out which investigated the displacement flow of viscoplastic materials. 
Below, we briefly review the previous work which studied the displacement flow of a non-Newtonian fluid by another Newtonian/non-Newtonian fluid.

Dimakopoulos and Tsamopoulos [28] studied the displacement of a viscoplastic material by air in straight and suddenly constricted tubes. They have shown that unyielded material arises in front of the air bubble and in the case of a constricted tube, near the recirculation corner. Papaioannou et al. [29], on the other hand, have studied the displacement of air by a viscoplastic fluid and revealed the conditions for the detachment of the viscoplastic material from the solid wall. Allouche et al. [30] and Wielage-Burchard et al. [31] studied the displacement flow of Bingham fluid by another fluid of same density in a plane channel. As the finger penetrates inside the channel a static residual layer of the displaced fluid is left behind the finger. They investigated the thickness of this residual layer for different Bingham numbers and compared their results with those obtained using the lubrication approximation.

The use of the discontinuous Bingham model for modeling the viscoplastic behaviour is not trivial because the yield surface is not known $a$ priori but must be determined as part of the solution. Generally, viscosity regularisation methods can be used with caution in order to overcome this difficulty. Frigaard and Nouar [32] studied the effects of different viscosity regularisation models, such as the simple model [30], the Bercovier and Engleman model [33] and the Papanastasiou model [34] on the flow dynamics and found that the latter model performs better than the other 
two models. However, Frigaard and Nouar [32] remarked that the regularization methods should be used carefully in flow configurations, such as thin-film flows, by choosing very small values of the regularising parameter.

Most of the numerical studies concerning displacement flows in the above review are for miscible systems, but few computational studies have been carried out on immiscible systems. Numerical simulation of immiscible systems are expensive computationally due to the presence of sharp interfacial dynamics. During the past few decades, lattice Boltzmann method (LBM) has emerged as a promising technique for multiphase flow simulations [35]. The LBM is a mesoscopic model of fluid flows, which has its origins in kinetic gas theory. In the LBM, components of velocity and density are calculated by taking the moments of the distribution functions. It is a simple and elegant method having several other advantages, such as being easy to implement, with no need to resolve the interface explicitly, and massive parallel efficiency. The LBM involves only three explicit steps: (i) collision, (ii) streaming, and (ii) calculation of variables. The most time-consuming step in any conventional Navier-Stokes solver, solution of the pressure Poisson equation is not there in LBM, which increases the computational efficiency of this method. Based on the class of problem of interest, researchers have been using different LBM approaches for multiphase flows, mainly, the color segregation method of Gunstensen et al. [36], method of Shan and Chen [37], the free energy approach of Swift et al. 
[38] and the method of He and co-workers [39-41]. Using the method of Shan and Chen [37], the displacement flow of two immiscible liquids have been studied by several researchers [14-17]. The Reynolds number considered in these studies are very low, thus they did not observe any interfacial instabilities. Recently, Redapangu et al. [18] investigated the displacement flow of two immiscible Newtonian liquids at moderate Reynolds number using the method of He et al. [39]. They investigated the effects of the Atwood number, viscosity ratio, and angle of inclination on the flow dynamics and observed sawtooth-type waves at the interface separating the liquids. Also the lattice Boltzmann method has been used for viscoplastic fluid flows (see for examples Vikhansky [42, 43] and Derksen [44]).

The buoyant displacement flow of one fluid by another fluid has been studied by several researchers (see [18] and references therein) and displacement flow of miscible viscoplastic fluids without density contrast has been studied by Frigaard and co-workers [30, 31] as discussed above. Also as they were interested in investigating mud removal in the primary cementing of oil-gas well bore, they considered isodensity fluids in their studies. In the present work, the pressure-driven displacement flow of two immiscible liquids of different densities and viscosities is studied using a multiphase lattice Boltzmann method $[39,45]$. In order to achieve high computational efficiency, our LBM algorithm is implemented on a graphics processing unit (GPU) [46]. It is also important to note here that the work 
of Dimakopoulos and Tsamopoulos [28] and Papaioannou et al. [29] are restricted to air/viscoplastic material systems, whereas Wielage-Burchard et al. [31] is for density-matched materials. Our work provides a generalisation of these studies and considers a different parameter range. Another important focus here is on the development of the LBM which is used to study the 2D problem first. This versatile, and massively-parallelisable method can then be extended readily to study the fully-3D problem, and to even include the effects of turbulence.

The rest of the paper is organized as follows. The details of the problem formulation and the LBM approach used to carry out the computations are provided in Section 3; the results are discussed in Section 3, and concluding remarks are given in Section 4.

\section{Formulation}

We consider the pressure-driven displacement of a viscoplastic, incompressible fluid of viscosity $\mu_{2}$ and density $\rho_{2}$ (fluid '2') initially filled inside a horizontal two-dimensional channel. A Newtonian fluid (fluid '1') of viscosity $\mu_{1}$ and density $\rho_{1}$ is injected from the inlet through an imposed pressure-gradient, as shown in Fig. 1. A rectangular coordinate system $(x, y)$ is used to model the flow dynamics, where $x$ and $y$ denote the coordinates in the horizontal and the wall-normal directions, respectively. The channel inlet and outlet are located at $x=0$ and $L$, respectively. The rigid and impermeable walls of the channel are located at $y=0$ and $H$, 
respectively. The aspect ratio of the channel, $L / H$, is $48 . g$ is the acceleration due to gravity acting in the negative $y$-direction.

\subsection{Numerical method}

The two-phase lattice Boltzmann method used in the present study is similar to that of He and co-workers [39-41]. Previously, Sahu and Vanka [45] modified this approach in order to account for unequal dynamic viscosity of the fluids and studied buoyancy-driven flow in an inclined channel. Recently, Redapangu et al. [18] studied pressure-driven displacement flow of Newtonian fluids using the same approach. The methodology is briefly described below.

Two evolution equations for the index distribution function (f) and the pressure distribution function $(\mathrm{g})$ are given by:

$$
\begin{gathered}
\mathrm{f}_{\alpha}\left(\mathbf{x}+\mathbf{e}_{\alpha} \delta \mathrm{t}, \mathrm{t}+\delta \mathrm{t}\right)-\mathrm{f}_{\alpha}(\mathbf{x}, \mathrm{t})=-\frac{\mathrm{f}_{\alpha}(\mathbf{x}, \mathrm{t})-\mathrm{f}_{\alpha}^{\mathrm{eq}}(\mathbf{x}, \mathrm{t})}{\tau}-\frac{2 \tau-1}{2 \tau} \frac{\left(\mathbf{e}_{\alpha}-\mathbf{u}\right) \cdot \nabla \psi(\phi)}{c_{\mathrm{s}}^{2}} \Gamma_{\alpha}(\mathbf{u}) \delta \mathrm{t}, \\
\mathrm{g}_{\alpha}\left(\mathbf{x}+\mathbf{e}_{\alpha} \delta \mathrm{t}, \mathrm{t}+\delta \mathrm{t}\right)-\mathrm{g}_{\alpha}(\mathbf{x}, \mathrm{t})=-\frac{\mathrm{g}_{\alpha}(\mathbf{x}, \mathrm{t})-\mathrm{g}_{\alpha}^{\mathrm{eq}}(\mathbf{x}, \mathrm{t})}{\tau}+ \\
\frac{2 \tau-1}{2 \tau}\left(\mathbf{e}_{\alpha}-\mathbf{u}\right) \cdot\left[\Gamma_{\alpha}(\mathbf{u})\left(F_{s}+\mathbf{G}\right)-\left(\Gamma_{\alpha}(\mathbf{u})-\Gamma_{\alpha}(0)\right) \nabla\left(p-c_{s}^{2} \rho\right)\right] \delta t, \quad(2)
\end{gathered}
$$

where

$$
\Gamma_{\alpha}(\mathbf{u})=t_{\alpha}\left[1+\frac{\mathbf{e}_{\alpha} \cdot \mathbf{u}}{c_{s}^{2}}+\frac{\left(\mathbf{e}_{\alpha} \cdot \mathbf{u}\right)^{2}}{2 c_{s}^{4}}-\frac{\mathbf{u}^{2}}{2 c_{s}^{2}}\right] .
$$

Here $\mathbf{u}=(u, v)$ represents the two-dimensional velocity field; $u$ and $v$ denote velocity components in the $x$ and $y$ directions, respectively; $\delta t$ is the time step; $\tau$ is the single relaxation time using the Bhatnagar-Gross-Krook (BGK) model [47]. The kinematic viscosity, $\nu$ is related to the relaxation 
time as $\nu=(\tau-1 / 2) \delta t c_{s}^{2}$, where $c_{s}^{2}=1 / 3$. The corresponding macroscopic equations describing the evolution equations [Eqs. (1) and (2)] can be found in Zhang et al. [39].

The evolution equations are simulated with a two-dimensional nine-velocity model (D2Q9), where

$$
\mathbf{e}_{\alpha}=\left\{\begin{array}{cc}
0, & \alpha=0 \\
{\left[\cos \left(\frac{(\alpha-1) \pi}{2}\right), \sin \left(\frac{(\alpha-1) \pi}{2}\right)\right],} & \alpha=1,2,3,4 \\
\sqrt{2}\left[\cos \left(\frac{(\alpha-5) \pi}{2}+\frac{\pi}{4}\right), \sin \left(\frac{(\alpha-5) \pi}{2}+\frac{\pi}{4}\right)\right], & \alpha=5,6,7,8 .
\end{array}\right.
$$

The weighing coefficients, $t_{\alpha}$ are given by:

$$
t_{\alpha}=\left\{\begin{array}{rc}
4 / 9, & \alpha=0 \\
1 / 9, & \alpha=1,2,3,4 \\
1 / 36, & \alpha=5,6,7,8 .
\end{array}\right.
$$

Here $\alpha$ is the number which indicates the position of the node in the lattice. The equilibrium distribution functions, $\mathrm{f}_{\alpha}^{e q}$ and $\mathrm{g}_{\alpha}^{e q}$ are given by

$$
\begin{aligned}
& \mathrm{f}_{\alpha}^{e q}=t_{\alpha} \phi\left[1+\frac{\mathbf{e}_{\alpha} \cdot \mathbf{u}}{c_{s}^{2}}+\frac{\left(\mathbf{e}_{\alpha} \cdot \mathbf{u}\right)^{2}}{2 c_{s}^{4}}-\frac{\mathbf{u}^{2}}{2 c_{s}^{2}}\right] \text { and } \\
& \mathrm{g}_{\alpha}^{e q}=t_{\alpha}\left[p+\rho c_{s}^{2}\left(\frac{\mathbf{e}_{\alpha} \cdot \mathbf{u}}{c_{s}^{2}}+\frac{\left(\mathbf{e}_{\alpha} \cdot \mathbf{u}\right)^{2}}{2 c_{s}^{4}}-\frac{\mathbf{u}^{2}}{2 c_{s}^{2}}\right)\right]
\end{aligned}
$$

The index function $(\phi)$, pressure $(p)$ and velocity field $(\mathbf{u})$ are calculated using:

$$
\begin{gathered}
\phi=\sum \mathrm{f}_{\alpha}, \\
p=\sum \mathrm{g}_{\alpha}-\frac{1}{2} \mathbf{u} \cdot \nabla \psi(\rho) \delta t
\end{gathered}
$$




$$
\rho \mathbf{u} c_{s}^{2}=\sum \mathbf{e}_{\alpha} \mathrm{g}_{\alpha}+\frac{c_{s}^{2}}{2}\left(\mathbf{F}_{\mathbf{s}}+\mathbf{G}\right) \delta \mathrm{t}
$$

The fluid density and kinematic viscosity are calculated from the index function as:

$$
\begin{aligned}
\rho(\phi) & =\rho_{1}+\frac{\phi-\phi_{1}}{\phi_{2}-\phi_{1}}\left(\rho_{2}-\rho_{1}\right), \\
\nu(\phi) & =\nu_{1} \exp \left[\frac{\phi-\phi_{1}}{\phi_{2}-\phi_{1}} \ln \left(\frac{\nu_{2}}{\nu_{1}}\right)\right],
\end{aligned}
$$

where $\nu_{1}$ and $\nu_{2}$ are the kinematic viscosities of fluid ' 1 ' and ' 2 ', respectively. $\phi_{1}$ and $\phi_{2}$ are minimum and maximum values of the index function; in the present study $\phi_{1}$ and $\phi_{2}$ are given values of 0.02381 and 0.2508 , respectively [39].

We use the following expression of $\psi(\phi)$ using the Carnahan-Starling fluid equation of state which describes the process of phase separation for non-ideal gases and fluids [48-52]:

$$
\psi(\phi)=c_{s}^{2} \phi\left[\frac{1+\phi+\phi^{2}-\phi^{3}}{(1-\phi)^{3}}-1\right]-a \phi^{2},
$$

where $a$ determines the strength of molecular interactions. The critical value of Carnahan-Starling equation of state, $a_{c}=3.53374$. If $a>a_{c}$ both the fluids will remain immiscible, however, it should be noted that very large values of $a$ can lead to loss of convergence. Here we have chosen $a$ to be 4 in the present study [39]. The gradient of $\psi(\phi)$ describes the physical intermolecular interactions for non-ideal gases or dense fluids. This term plays a key role in separating the phases [41]. 
We use the Herschel-Bulkley model in order to describe the flow of the viscoplastic material, which is being displaced by a Newtonian fluid injected at the inlet of the channel. There are three commonly used regularized non-Newtonian fluid models available in the literature [32], which are given by:

$$
\begin{gathered}
\mu_{2}=\mu_{0}\left(\Pi+\epsilon_{d}\right)^{n-1}+\frac{\tau_{0}}{\Pi+\epsilon_{d}}, \\
\mu_{2}=\mu_{0}\left(\Pi+\epsilon_{d}\right)^{n-1}+\frac{\tau_{0}}{\sqrt{\Pi^{2}+\epsilon_{d}^{2}}}, \\
\mu_{2}=\mu_{0}\left(\Pi+\epsilon_{d}\right)^{n-1}+\tau_{0}\left(\frac{1-e^{-N \Pi}}{\Pi}\right),
\end{gathered}
$$

where $\tau_{0}$ is the yield shear stress; $\Pi \equiv\left(2 E_{i j} E_{i j}\right)^{1 / 2}$ represents the second invariant of the strain-rate tensor, $E_{i j}=\frac{1}{2}\left(\partial u_{i} / \partial x_{j}+\partial u_{j} / \partial x_{i}\right)$, where $i, j$ correspond to the coordinates; $n$ is the power-law flow index of the fluid. $\mu_{0}$ is the flow consistency index (this is same as the viscosity of fluid 2 when $\tau_{0}=0$ and $\left.n=1\right) . N$ is the stress growth exponent and for $n=1$, it is equivalent to $\epsilon_{d}^{-1}$. We will refer to eqs. (14), (15) and (16) as the 'simple regularized viscosity model' [30], Bercovier and Engleman's model [33] and Papanastasiou's model [34], respectively.

The surface tension $\left(F_{s}\right)$ and gravity $(\mathbf{G})$ forces are given by

$$
F_{s}=\kappa \phi \nabla \nabla^{2} \phi, \quad \text { and } \quad \mathbf{G}=\left(\rho-\rho_{\mathrm{m}}\right) g,
$$

where $\kappa$ is the magnitude of surface tension and $\rho_{m} \equiv\left(\rho_{1}+\rho_{2}\right) / 2$. The 
surface tension, $\sigma$ can be related to $\kappa$ as follows [53]:

$$
\sigma=\kappa \int\left(\frac{\partial \phi}{\partial \zeta}\right)^{2} d \zeta
$$

where $\zeta$ is the direction normal to the interface [39].

The hydrodynamic boundary conditions based on the ghost fluid approach are used to simulate the boundaries (implementation of no-slip boundary conditions at the walls) and equilibrium distribution functions [45]. A Neumann boundary condition for the pressure is used at the outlet, while the constant volumetric flow rate condition, $(u, v)=\left(-6 Q\left(y^{2} / H^{2}-y\right), 0\right)$, is imposed at the inlet. Here, $Q$ is the total flow rate per unit length in the spanwise direction. A fourth order compact scheme is used to discretize $\nabla \psi[54]$.

The various dimensionless parameters describing the flow characteristics are the Atwood number, $A t\left(\equiv\left(\rho_{2}-\rho_{1}\right) /\left(\rho_{2}+\rho_{1}\right)\right)$, the Reynolds number, $R e\left(\equiv Q \rho_{1} / \mu_{1}\right)$, the Bingham number, $B n\left(\equiv \tau_{0} H^{2} / \mu_{1} Q\right)$, the Richardson number, $R i\left(\equiv g H^{3} / Q^{2}\right)$, the viscosity ratio, $m=\mu_{0} / \mu_{1}$, and dimensionless viscosity regularization parameter, $\epsilon=Q \epsilon_{d} / H^{2}$. The dimensionless time is defined as $t=H^{2} / Q$. To accelerate the computational efficiency, the algorithm is implemented on a Graphics Processing Unit (GPU). Our GPU based multiphase lattice Boltzmann solver using the double-precision variable provides a speed-up factor of 12 as compared to a corresponding CPU based solver $[46,55]$. 


\section{Results and discussion}

We begin the presentation of our results by conducting a grid convergence test. In Fig. 2 (a), (b) and (c), the spatio-temporal evolution of the contours of the index function, $\phi$ are shown for grids $3168 \times 66$, $4704 \times 98$ and $6240 \times 130$, respectively, for $R e=100, A t=0.2, R i=0.1$, $m=2, \kappa=0, B n=10, n=1.1$ and $\epsilon=10^{-9}$. The simple regularized viscosity model is used to generate this plot. The parameter values used in generating this figure correspond to a situation where a highly viscous, denser non-Newtonian fluid (fluid '2') is displaced by a lighter, Newtonian fluid of lower viscosity (fluid ' 1 '). In general, the flow is expected to be destabilized because of the density and viscosity contrast, via a Rayleigh-Taylor or a Kelvin-Helmholtz (KH) type instability. It can be seen that due to the imposed pressure-gradient a 'finger' of the less viscous lighter fluid penetrates into the bulk of the more viscous, denser fluid. The finger is symmetrical at early times, but becomes asymmetrical at later times due to the gravity force acting in the vertically downward direction. At the edge of the trailing film, instabilities of sawtooth-like shape arise, due to a KH instability, and are being convected downstream. The interfacial waves resulting from the instabilities at the downstream portion of the channel (obtained using $6240 \times 130$ grid at $t=50$ ) are shown as the inset at the bottom of Fig. 2. The flow dynamics obtained using the different grids exhibit some minor quantitative variations upon mesh-refinement. However, as it will be shown below that there is very 
good convergence with respect to the layer thickness. In addition, we have conducted a linear stability analysis (similar to the one presented in [18]) in a core-annular configuration by specifying the thickness of the residual layer obtained from the numerical simulations, details of the analysis are given in the appendix. It can be seen in Fig. 3 that the wavelength of the most dangerous mode in the linear stability analysis is in excellent agreement with that of the interfacial waves seen in Fig. 2.

In Fig. 4 (a) and (b), we plot the temporal variation of the dimensionless volume of fluid '2', $M_{t} / M_{0}$, and the average residual thickness of the bottom layer, $\bar{h}$, for the same values the as those used to generate Fig. 2. Here, $M_{t}=\int_{0}^{L} \int_{0}^{H} \frac{\phi-\phi_{1}}{\phi_{2}-\phi_{1}} d y d x, M_{0}$ denotes the volume of fluid ' 2 ' initially occupying the channel $\left(M_{0}=\frac{\phi-\phi_{1}}{\phi_{2}-\phi_{1}} L H\right)$, and $\bar{h}=\frac{1}{x_{l}-x_{t}} \int_{0}^{H / 2} \int_{x_{t}}^{x_{l}} \frac{\phi-\phi_{l}}{\phi_{h}-\phi_{l}} d x d y$, where in, $x_{l}$ and $x_{t}$ are the position of the leading and trailing edges of the finger, respectively. It can be seen in Fig. $4(\mathrm{a})$ that $M_{t} / M_{0}$ undergoes an almost linear decrease due the displacement of fluid ' 2 ' by fluid ' 1 '. It can also be observed that slope of $M_{t} / M_{0}$ versus time plot is steeper than that of the plug flow line, given by $M_{t} / M_{0}=1-t H / L$ (shown by the dotted line in Fig. 4(a)).

It can be seen in Fig. 4(b) that the height of the residual bottom layer remains almost constant throughout the simulation except for very early times. Moreover, it is shown that the difference in the results obtained using $4704 \times 98$ and $6240 \times 130$ grids are very small and the latter grid has been used for generating the rest of the results presented in this paper. It 
should be noted here that the present code has been validated extensively by comparing with other experimental studies of buoyancy-driven flows. We have also performed finite-volume simulation for this configuration and compared the results obtained from both approaches. This has been reported in our previous paper [45].

The thickness of this residual layer, and the removal time, was also previously studied by Frigaard and co-workers [30, 31] for low Reynolds number flows. As a part of the validation exercise, we also compared the thickness of the residual layer obtained from the present simulation with that of Wielage-Burchard \& Frigaard [31] by setting $B n=20, m=1$, $A t=0$ and $\kappa=0$ in our code. We found that the values of the residual thickness for $R e=100$ and 200 are 0.15 and 0.14 , respectively. The shape of the predicted interface as well as the evaluated thickness of the residual layer are in excellent agreement with the results of Wielage-Burchard \& Frigaard [31]. It is to be noted here that the instabilities seen in Fig. 2 are due to the non-zero Atwood number considered in the present study.

Next, we investigate the effects of viscosity regularization parameter $(\epsilon)$ in the simple viscosity regularized model (given by Eq. 14) on the flow dynamics by plotting the spatio-temporal evolution of the $\phi$ contours for different values of $\epsilon$. The rest of the parameter values are $R e=100, R i=1$, At $=0.2, m=2, \kappa=0.0075, B n=30$ and $n=1.1$. As discussed by Frigaard and Nouar [32], the discontinuous Bingham model can be regularized by adding a small numerical parameter $\epsilon$ to the second invariant 
of the strain-rate tensor in order to avoid the singularity in the low shear region. It can be seen in Fig. 5 that the flow dynamics looks qualitatively similar for $10^{-6} \leq \epsilon \leq 10^{-12}$. Please note that we have tested the results for $n<1$ and found the same conclusions. The lowest value for $\epsilon$ increases the stiffness of the system of partial differential equations and thus we have used $\epsilon=10^{-9}$ to generate the rest of the results in this paper. Inspection of Fig. 5 also reveals that the sawtooth shape interfacial instabilities which was observed in Fig. 2 did not appear in this case. On the other hand, we notice that a few drops of the non-Newtonian fluid arise in the middle of the channel. Also as $R i=1$ in this case, the flow becomes more asymmetrical as compared to that in Fig. $2(R i=0.1)$.

Then, we proceed with the investigation of the effects of various viscosity regularized models [given by Eqs. (14)-(16)] proposed in literature (see for instance Ref. [32]) on the flow dynamics. This has been carried out to investigate the effects of these models in the framework of lattice Boltzmann method. In Fig. 6(a), (b) and (c), we present the spatio-temporal contours of the index function obtained using the simple model, Bercovier and Engleman's model and Papanastasiou's model, respectively for the parameter values $R e=100, R i=0.5, A t=0.2, m=2, \kappa=0.0075$, $B n=30$ and $n=1.1$. We also plot in Fig. 7 the spatio-temporal evolution of the unyielded domains (shown in black) obtained using the models above for the same parameter values as those used in Fig 5. The unyielded domain is the region where shear stress, $\tau \leq \tau_{0}$. It can be seen that the 
black region in the downstream (just after the finger) is the unyielded region which opposes the motion of the 'finger' of fluid ' 1 ' into the bulk of fluid '2'. Close inspection of Fig. 7 and the enlarged view of the region marked by rectangles, shown at the bottom of each panels, reveals that the thin region just above the interface separating the fluids and the drops of fluid '2' which appear inside the finger are also surrounded by unyielded material. This effect will be discussed below. It can be observed that the thickness of the residual layer, the small scale structures and location of the yield surface obtained using all rheological models match very well for the set of parameter values considered. Frigaard and Nouar [32] showed that for strain rates close to zero (i.e. when a material is stationary) the result obtained from Papanastasiou's model is closer to the theoretical prediction. However, for the flow in hand and for the particular selection of $\epsilon$ no significant differences are found and therefore we prefer to use, for the rest of this study, the simple regularized model since it is easier to implement.

Next, we investigate the effects of $B n$ number on the flow dynamics. The contours of the index function, $\phi$ at $t=20$ and $t=30$ are shown for three values of Bingham number in Fig. 8. The rest of the parameter values are $R e=100, A t=0.2, R i=1, \kappa=0.0075, m=2$ and $n=1$. The value of the flow index, $n$ is set equal to 1 in order to isolate the effects of $B n$ on the flow dynamics. The results shown in Fig. 8(a) are associated with the case when fluid ' 2 ' is also Newtonian. It can be seen in Fig. 8 (a) that as the finger of fluid ' 1 ' penetrates inside the channel, the upper elongated region of the 
finger becomes unstable, and a sawtooth shape wave is clearly visible at the later time. Close inspection of the contours at $t=20$ reveals that this wave originates at early times $(t \approx 20)$. When the fluid ' 2 ' is non-Newtonian it can be seen in panels (b), (c) and (d) of Fig. 8 that the width of the finger increases with increasing $B n$. This is due to the presence of the unyielded region at the front of the finger (shown in Fig. 9 for $B n=50$ ). It is also shown that the shear stress in this region decreases with increasing $B n$, which in turn decreases the velocity of the tip of the finger (this is evident in Fig. 8). However, for $B n=0$ it can be seen that the velocity of the finger tip is slightly lower than that for $B n=20$. An explanation for this is as follows: in the Newtonian case, there are no unyielded regions, but for any finite $B n$ the residual layers become unyielded (see inset at the bottom of Fig. 9). This creates a three-layer configuration, where the viscosity of the fluid in the near wall region increases as compared to that of the Newtonian fluid displacement. This increases the fluid velocity in the core region in case of non-Newtonian fluid with low $B n$, but as the $B n$ increases the unyielded region at the front of the finger becomes an important factor, which decreases the velocity of the finger tip (see Fig. 10). The presence of the unyielded material in the residual film leads to the suppression of the interfacial instability at higher $B n$.

In Fig. 11 (a), (b) and (c), we plot temporal variation of volume fraction of the displaced fluid $\left(M_{t} / M_{0}\right)$, the displacement rate of 'fluid '2', given by $\left(M_{t} / M_{0}\right)^{\prime}$, where prime represents the differentiation with respect to time, 
and the average residual thickness of the bottom layer, $\bar{h}$, respectively for different values of $B n$. It can be seen in Fig. 11 (a) and (b) that the effects of $B n$ is non-monotonic. The displacement rate increases with increasing the value of $B n$ upto $B n \approx 30$, but, further increase in $B n$ decreases the displacement rate. This is probably due to the formation of three-layer structure discussed above. It can be seen in Fig. 11 (b) that increasing the value of $B n$ increases the average residual thickness of the bottom layer. The thickness of the residual layer at the bottom is more than that at the top. The viscosity of this material increases with increasing Bingham number and becomes unyielded (as shown in Fig. 9). Thus this residual material becomes increasingly difficult to be removed for higher values of $B n$.

Finally, we investigate the effects of the flow index, $n$. In Figs. 12 and 13, the contours of the index function, $\phi$ and the unyielded domains (shown in black), and contours of the axial velocity, $u$ are plotted, respectively at $t=20$ and $t=30$ for different values of $n$. The rest of the parameters are $R e=100, A t=0.2, R i=1, \kappa=0.0075, m=2$ and $B n=30$. Here decreasing the value of $n$ reflects an increase in the shear-thinning tendency of the non-Newtonian fluid. It can be seen that for $n=0.7$ (i.e, for shear thinning fluid) the interfacial instability becomes vigorous. In this case, there is a competition between the effects created by the Bingham number with that of the shear thinning. For $n=0.7$ the unyielded material is absent in the region in front of the finger for the set of parameter values 
considered. Thus the finger penetrates freely inside the channel. For $n=1.3$ the effects of Bingham number and the flow index reinforce one another, i.e. to decrease the shear stress in the flow region. The rate of displacement, $\left(M_{t} / M_{0}\right)^{\prime}$, and the average residual thickness of the bottom layer, $\bar{h}$, for different values of $n$ are shown in Fig. 14. It can be observed in Fig. 14 (a) that the disappearance of the unyielded material due to the shear thinning behaviour of the fluid (decreasing the value of $n$ ) makes it easier for the fluid to penetrate inside the channel, thus leading to faster displacement. In Fig. 14 (b), it can be seen that the average residual thickness of the bottom layer, $\bar{h}$ increases almost linearly with time and decreases with increasing the value of $n$. Thus increasing the value of $n$ increases the unyielded region in the downstream of the channel, which in turn decreases the velocity of the finger tip. As expected, it was found (not shown) that the instabilities associated with different values of $n$ for $B n=0$ are more vigorous than those shown in Fig. 12 (for $B n=30$ ).

\section{Summary}

The pressure-driven displacement flow of a non-Newtonian fluid by a Newtonian fluid in a two-dimensional channel is investigated via a multiphase lattice Boltzmann method using the Carnahan-Starling equation of state. This method was originally proposed by He and co-workers [39-41] and recently used by many researchers $[45,51]$. This method uses two distribution functions in order to evaluate the flow variables, hydrodynamic 
pressure and the index function. The index function is used to distinguish both the fluids. We used three models for the non-Newtonian fluid, namely, a simple regularized model, the Bercovier and Engleman's model [33] and Papanastasiou's model [34]. The lattice Boltzmann predictions are validated against the results of linear stability theory and finite volume simulations. It was found that for the parameter values considered in this study all the models give very similar results. The effects of the Bingham number (which characterises the behaviour of the yield-stress of the fluid) and the flow index (which reflects the shear-thinning tendency of the fluid) are studied. It is shown that the rate of displacement depends non-monotonically on the viscoplasticity of the material. In addition, it is shown that increasing the Bingham number and the flow index increases the size of the unyielded region ahead of the displacing fluid and the residual layer adjacent to the walls. This in turn decreases the interfacial instabilities and the speed of the propagating finger.

\section{Acknowledgements}

KS thank the Department of Science and Technology, India for their financial support (Grant No: SR/FTP/ETA-85/2010). We also acknowledge Prof. Pratap Vanka (University of Illinois at Urbana-Champaign, USA) for the collaboration during the code development.

\section{Appendix: Linear stability analysis}


As the elongated 'finger' of the injected Newtonian fluid enters into the bulk of non-Newtonian fluid, a three-layer structure is formed inside the channel. A linear stability analysis is conducted for this idealised flow. The governing linear stability equation and boundary conditions are provided in this section. The readers are refereed to Redapangu et al. [18] for the relevant details. Applying a symmetry boundary condition at the centreline of the channel, the bottom part of the channel is only considered for this analysis. The fully-developed velocity profile for the basic state is assumed, i.e. streamwise velocity of the residual layer, $U_{1}=U_{1}(y)$, the Newtonian layer, $U_{2}=U_{2}(y)$, and vertical velocity, $V=0$ in both the fluids.

Each flow variable is expressed as the sum of a base state and a 2D perturbation:

$$
\left(u_{k}, v_{k}, p_{k}\right)(x, y, t)=\left(U_{k}, 0, P\right)(y)+\left(\hat{u}_{k}, \hat{v}_{k}, \hat{p}_{k}\right)(x, y, t)
$$

with $(k=1,2)$. Similarly $h$ and the viscosity of the non-Newtonian fluid, $\mu_{2}$ can be expanded as follows

$$
\begin{gathered}
h(x, t)=h^{0}+\hat{h}(x, t), \\
\mu_{2}(\pi)=\mu_{2}^{0}+\left.\frac{\partial \mu_{2}}{\partial \pi}\right|^{0}(\pi-\Pi)=\mu_{2}^{0}+\beta \hat{\pi},
\end{gathered}
$$

where the superscript ' 0 ' designates base state quantities, $\beta=(n-1) m \Pi^{n-2}-B n \Pi^{-2}$. By following the usual procedure $[18,22]$, the linearised stability equations are derived. These are re-expressed in terms of the stream-function, $\left(u_{k}, v_{k}\right)=\left(\partial \Psi_{k} / \partial y,-\partial \Psi_{k} / \partial x\right)(k=1,2)$ and the 
perturbation variables are decomposed using a normal-mode analysis, such as $\Psi_{k}(x, y, t)=\Phi_{k}(y) e^{\mathrm{i}(\alpha x-\omega t)}$, wherein $\Phi_{k}$ is the amplitude of the streamfunction, $\alpha$ and $\omega$ are the real wavenumber and complex frequency of the disturbance.

The linear stability equations are given by

$$
\begin{gathered}
\mathrm{i} \alpha r \operatorname{Re}\left[\left(\Phi_{2}^{\prime \prime}-\alpha^{2} \Phi_{2}\right)\left(U_{2}-c\right)-\Phi_{2} U_{2}^{\prime \prime}\right]=\mu_{2}^{0}\left[\Phi_{2}^{\prime \prime \prime \prime}-2 \alpha^{2} \Phi_{2}^{\prime \prime}+\alpha^{4} \Phi_{2}\right]+\beta U_{2}^{\prime} \pi^{\prime \prime}+ \\
2 \beta U_{2}^{\prime \prime} \pi^{\prime}+\beta U_{2}^{\prime \prime \prime} \pi+\alpha^{2} \beta U_{2}^{\prime} \pi-2 \alpha^{2} \mu_{2}^{0^{\prime}} \Phi_{2}^{\prime}+\mu_{2}^{0^{\prime \prime}} \Phi_{2}^{\prime \prime}+2 \mu_{2}^{0^{\prime}} \Phi_{2}^{\prime \prime \prime}+ \\
\beta^{\prime \prime} U_{2}^{\prime} \pi+2 \beta^{\prime} U_{2}^{\prime} \pi^{\prime}+2 \beta^{\prime} U_{2}^{\prime \prime} \pi+\mu_{2}^{0 \prime \prime} \alpha^{2} \Phi_{2} \\
\mathrm{i} \alpha \operatorname{Re}\left[\left(\Phi_{1}^{\prime \prime}-\beta^{2} \Phi_{1}\right)\left(U_{1}-c\right)-\Phi_{1} U_{1}^{\prime \prime}\right]=\left[\Phi_{1}^{\prime \prime \prime \prime}-2 \beta^{2} \Phi_{1}^{\prime \prime}+\beta^{4} \Phi_{1}\right]
\end{gathered}
$$

Here, the prime represents differentiation with respect to $y$ and $r$ represents the density ratio, $\rho_{2} / \rho_{1}$. In the temporal stability analysis considered in this section, $\omega_{i}>0$ indicates the presence of a linear instability.

The solution of Eqs. (22) and (23) subject to the following boundary conditions: the no-slip and no-penetration conditions at the bottom wall:

$$
\Phi_{2}=\Phi_{2}^{\prime}=0
$$

and

$$
\Phi_{1}^{\prime}=\Phi_{1}^{\prime \prime \prime}=0
$$

at the centreline. Using the continuity of the velocity and stress components for the disturbance in the axial and the wall-normal directions 
at the interface, along with the kinematic boundary condition, we obtained:

$$
\begin{gathered}
\Phi_{1}=\Phi_{2}, \\
\Phi_{1}^{\prime}-\Phi_{2}^{\prime}+\frac{\Phi_{1}}{\left(c-U_{1}\right)}\left(U_{1}^{\prime}-U_{2}^{\prime}\right)=0 \\
\mu_{2}^{0}\left(\Phi_{2}^{\prime \prime}+\alpha^{2} \Phi_{2}\right)-\left(\Phi_{1}^{\prime \prime}+\alpha^{2} \Phi_{1}\right)+\frac{\left(\mu_{2}^{0} U_{2}^{\prime \prime}-U_{1}^{\prime \prime}\right)}{\left(U_{1}-c\right)}+\mathrm{i} \alpha \beta U_{2}^{\prime} \pi=0, \\
\alpha r R e\left[\Phi_{2}^{\prime}\left(c-U_{2}\right)+\Phi_{2} U_{2}^{\prime}\right]-\alpha \operatorname{Re}\left[\Phi_{1}^{\prime}\left(c-U_{1}\right)+\Phi_{1} U_{1}^{\prime}\right]+2 \mathrm{i} \alpha^{2}\left(\mu_{2}^{0} \Phi_{2}^{\prime}-\frac{3}{2} \Phi_{1}^{\prime}\right)+ \\
2 \mathrm{i} \alpha^{2} \mu_{2}^{0} \Phi_{2}^{\prime}-i\left[\mu_{2}^{0}\left(\Phi_{2}^{\prime \prime \prime}+\alpha^{2} \Phi_{2}^{\prime}\right)+\mu_{2}^{0^{\prime}}\left(\Phi_{2}^{\prime \prime}+\alpha^{2} \Phi_{2}\right)+\beta U_{2}^{\prime} \pi^{\prime}+\beta^{\prime} U_{2}^{\prime} \pi+\beta U_{2}^{\prime \prime} \pi-\Phi_{1}^{\prime \prime \prime}\right] \\
=\left(\frac{\alpha^{2}}{C a}+\mathcal{G}\right) \mathrm{i} \alpha \frac{\left(\Phi_{1}^{\prime}-\Phi_{2}^{\prime}\right)}{\left(U_{2}^{\prime}-U_{1}^{\prime}\right)},
\end{gathered}
$$

where $\mathcal{G} \equiv\left(\rho_{2}-\rho_{1}\right) g H^{3} / \mu_{1} Q$. The above stability equations, along with the boundary conditions (given in Eqs. (22)-(29)) constitute an eigenvalue problem, which is solved using a spectral collocation method using a public domain software LAPACK. The linear stability solver are then validated by performing a grid-convergence test and by also comparing with the previous work on Newtonian and non-Newtonian fluids [22].

\section{References}

[1] S. M. Taghavi, K. Alba and T. Séon, K. Wielage-Burchard, D. M. Martinez, and I. A. Frigaard, Miscible displacement flows in near-horizontal ducts at low atwood number, J. Fluid Mech. 696 (2012) 175-214. 
[2] D. D. Joseph, R. Bai, K. P. Chen, Y. Y. Renardy, Core-annular flows, Ann. Rev. Fluid Mech. 29 (1997) 65-90.

[3] G. M. Homsy, Viscous fingering in porous media, Ann. Rev. Fluid Mech. 19 (1987) 271-311.

[4] R. Govindarajan, K. C. Sahu, Instabilities in viscosity stratified flow, Ann. Rev. Fluid Mech. (to appear, 2014)

[5] C.-Y. Chen, E. Meiburg, Miscible displacement in capillary tubes. part 2. numerical simulations, J. Fluid Mech. 326 (1996) 57-90.

[6] N. Rakotomalala, D. Salin, P. Watzky, Miscible displacement between two parallel plates: BGK lattice gas simulations, J. Fluid Mech. 338 (1997) 277-297.

[7] N. Goyal, E. Meiburg, Miscible displacements in Hele-Shaw cells: two-dimensional base states and their linear stability, J. Fluid Mech. 558 (2006) 329-355.

[8] P. Petitjeans, P. Maxworthy, Miscible displacements in capillary tubes. Part 1. Experiments, J. Fluid Mech. 326 (1996) 37-56.

[9] K. C. Sahu, H. Ding, P. Valluri, O. K. Matar, Prssure-driven miscible two-fluid channel flow with density gradients, Phys. Fluids 21 (2009) 043603. 
[10] S. M. Taghavi, T. Séon, D. M. Martinez, I. A. Frigaard, Buoyancy-dominated displacement flows in near-horizontal channels: the viscous limit, J. Fluid Mech. 639 (2009) 1-35.

[11] S. M. Taghavi, T. Séon, D. M. Martinez, I. A. Frigaard, Stationary residual layers in buoyant newtonian displacement flows, Phys. Fluids 23 (2011) 044105.

[12] M. Mishra, A. D. Wit, K. C. Sahu, Double diffusive effects on pressure-driven miscible displacement flow in a channel, J. Fluid Mech. 712 (2012) 579-597.

[13] D. D. Joseph, M. Renardy, Y. Y. Renardy, Instability of the flow of two immiscible liquids with different viscosities in a pipe, J. Fluid Mech. 141 (1984) 309-317.

[14] Q. Kang, D. Zhang, S. Chen, Immiscible displacement in a channel: simulations of fingering in two dimensions, Adv. Water Resour. 27 (2004) 13-22.

[15] J. Chin, E. S. Boek, P. V. Coveney, Lattice Boltzmann simulation of the flow of binary immiscible fluids with different viscosities using the - Shan-Chen microscopic interaction model, Phil. Trans. Math. Phys. Eng. Sci. 360 (2002) 547-558.

[16] P. Grosfils, J. P. Boon, J. Chin, Structural and dynamical 
characterization of Hele-Shaw viscous fingering, Phil. Trans. Math. Phys. Eng. Sci. 362 (2004) 1723-1734.

[17] B. Dong, Y. Y. Yan, W. Li, Y. Song, Lattice Boltzmann simulation of viscous fingering phenomenon of immiscible fluids displacement in a channel, Computers \& Fluids 39 (2010) 768-779.

[18] P. R. Redapangu, K. C. Sahu, S. P. Vanka, A study of pressure-driven displacement flow of two immiscible liquids using a multiphase lattice Boltzmann approach, Phys. Fluids 24 (2012) 102110.

[19] K. C. Sahu, H. Ding, P. Valluri, O. K. Matar, Linear stability analysis and numerical simulation of miscible channel flows, Phys. Fluids 21 (2009) 042104.

[20] C. S. Yih, Instability due to viscous stratification, J. Fluid Mech. 27 (1967) 337-352.

[21] S. G. Yiantsios, B. G. Higgins, Numerical solution of eigenvalue problems using the compound matrix-method, J. Comp. Phys. 74 (1988) 25-40.

[22] K. C. Sahu, O. K. Matar, Three-dimensional linear instability in pressure-driven two-layer channel flow of a Newtonian and a Herschel-Bulkley fluid, Phys. Fluids 22 (2010) 112103.

[23] R. Govindarajan, Effect of miscibility on the linear instability of two-fluid channel flow, Int. J. Multiphase Flow 30 (2004) 1177-1192. 
[24] B. Selvam, S. Merk, R. Govindarajan, E. Meiburg, Stability of miscible core-annular flows with viscosity stratification, J. Fluid Mech. 592 (2007) 23-49.

[25] S. V. Malik, A. P. Hooper, Linear stability and energy growth of viscosity stratified flows, Phys. Fluids 17 (2005) 024101.

[26] K. C. Sahu, R. Govindarajan, Linear stability of double-diffusive two-fluid channel flow, J. Fluid Mech. 687 (2011) 529-539.

[27] E. Lajeunesse, J. Martin, N. Rakotomalala, D. Salin, and Y. C. Yortsos, Miscible displacement in a Hele-Shaw cell at high rates, J. Fluid Mech. 398 (1999) 299-319.

[28] Y. Dimakopoulos, J. Tsamopoulos, Transient displacement of a viscoplastic material by air in straight and suddenly constricted tubes, Journal of Non-Newtonian Fluid Mechanics 112 (1) (2003) 43-75.

[29] J. Papaioannou, G. Karapetsas, Y. Dimakopoulos, J. Tsamopoulos, Injection of a viscoplastic material inside a tube or between two parallel disks: Conditions for wall detachment of the advancing front, Journal of Rheology 53 (5) (2009) 1155-1191.

[30] M. Allouche, I. A. Frigaard, G. Sona, Static wall layers in the displacement of two visco-plastic fluids in a plane channel, J. Fluid Mech. 424 (2000) 243-277. 
[31] K. Wielage-Burchard, I. A. Frigaard, Static wall layers in plane channel displacement flows, J. Non. Newt. Fluid Mech. 166 (2011) 245-261.

[32] I. A. Frigaard, C. Nouar, On the usage of viscosity regularisation methods for visco-plastic fluid flow computation, J. Non. Newt. Fluid Mech. 127 (2005) 1-26.

[33] M. Bercovier, M. Engleman, A finite-element method for incompressible non-Newtonian flows, J. Comput. Phys 36 (1980) $313-326$.

[34] T. C. Papanastasiou, Flows of materials with yield, J. Rheol 31(5) (1987) 385-404.

[35] S. Chen, G. D. Doolen, Lattice Boltzmann method for fluid flows, Ann. Rev. Fluid Mech. 30 (1998) 329-364.

[36] A. K. Gunstensen, D. H. Rothman, S. Zaleski, G. Zanetti, Lattice Boltzmann model for immiscible fluids, Phys. Rev. A. 43 (1991) 4320-4327.

[37] X. Shan, H. Chen, Lattice Boltzmann model for simulating flows with multiple phases and components, Phys. Rev. E. 47(3) (1993) $1815-1819$.

[38] M. R. Swift, W. R. Osborn, J. M. Yeomans, Lattice-Boltzmann simulation of nonideal fluids, Phys. Rev. Lett. 75 (1995) 830-833. 
[39] R. Zhang, X. He, S. Chen, Interface and surface tension in incompressible lattice Boltzmann multiphase model, Computer Physics Communications 129 (2000) 121-130.

[40] X. He, R. Zhang, S. Chen, G. D. Doolen, On the three-dimensional Rayleigh-Taylor instability, Phys. Fluids 11(5) (1999) 1143-1152.

[41] X. He, S. Chen, R. Zhang, A lattice Boltzmann scheme for incompressible multiphase flow and its application in simulation of Rayleigh-Taylor instability, J. Comput. Phys 152 (1999) 642-663.

[42] A. Vikhansky, Lattice-boltzmann method for yield-stress liquids 155 (2008) 95-100.

[43] A. Vikhansky, Construction of lattice-boltzmann schemes for non-newtonian and two-phase flows, The Canadian journal of Chemical Engineering 90 (2012) 1081-1091.

[44] J. J. Derksen, Simulations of mobilization of bingham layers in a turbulently agitated tank 191 (2013) 25-34.

[45] K. C. Sahu, S. P. Vanka, A multiphase lattice Boltzmann study of buoyancy-induced mixing in a tilted channel, Computers \& Fluids 50 (2011) 199-215.

[46] Computational fluid dynamics using graphics processing units: Challenges and Opportunities, Proceedings of the ASME 2011 
International Mechanical Engineering Congress and Exposition, Denver, Colorado, USA, 2011.

[47] P. L. Bhatnagar, E. P. Gross, M. Krook, A model for collision process in gases. I. small amplitude processes in charged and neutral one-component system, Phys. Rev. 94 (1954) 511-525.

[48] N. F. Carnahan, K. E. Starling, Equation of state for non-attracting rigid spheres, J. Chem. Phys. 51 (1969) 635-636.

[49] K. N. Premnath, J. Abraham, Lattice Boltzmann model for axisymmetric multiphase flows, Phys. Rev. E. 71 (2005) 056706.

[50] Q. Chang, J. I. D. Alexander, Application of the lattice Boltzmann method to two-phase Rayleigh-Benard convection with a deformable interface, J. Comput. Phys 212 (2006) 473-489.

[51] A. Fakhari, M. H. Rahimian, Simulation of falling droplet by the lattice Boltzmann method, Communications in Nonlinear Science and Numerical Simulations 14 (2009) 3046-3055.

[52] A. Fakhari, M. H. Rahimian, Investigation of deformation and breakup of a moving droplet by the method of lattice Boltzmann equations, Int. J. Numer. Meth. Fluids 64 (2010) 827-849.

[53] R. Evans, The nature of the liquid-vapor interface and other topics in the statistical mechanics of non-uniform classical fluids, Adv. Phys 28 (1979) 143-200. 
[54] T. Lee, C.-L. Lin, A stable discretization of the lattice Boltzmann equation for simulation of incompressible two-phase flows at high density ratio, J. Comput. Phys 206 (2005) 16-47.

[55] P. R. Redapangu, S. P. Vanka, K. C. Sahu, Multiphase lattice Boltzmann simulations of buoyancy-induced flow of two immiscible fluids with different viscosities, European Journal of Mechanics B/Fluids 34 (2012) 105-114. 


\section{List of figures captions}

Fig. 1: Schematic showing the geometry (not to scale) and initial flow configuration. The inlet and outlet are located at $x=0$ and $x=L$, respectively. The aspect ratio of the channel, $L / H$, is 48 . Initially the channel is filled with fluids ' 1 ' and ' 2 ' from $0 \leq x \leq 5$ and $5 \leq x \leq L$ of the channel, respectively.

Fig. 2: Contours of the index function, $\phi$ for different mesh densities: (a) $3168 \times 66$, (b) $4704 \times 98$, (c) $6240 \times 130$. The rest of the parameters are $R e=100, A t=0.2, R i=0.1, m=2, \kappa=0, B n=10, n=1.1$ and $\epsilon=10^{-9}$. The inset at the bottom of each panel represents the enlarged view of the contours at $t=30$ obtained using respective gridsize.

Fig. 3: (a) Growth rate versus wave number of a infinitesimal disturbance obtained from our linear stability analysis, (b) enlarge view of the contours at $t=30$. The rest of the parameter values are the same as those used to generate Fig. 2. The wavelength of the interfacial mode observed in the linear stability analysis and LBM simulation are 1.54 and 1.49 , respectively. Fig. 4: (a) Temporal variation of volume fraction of the displaced fluid $\left(M_{t} / M_{0}\right)$, (b) the average residual thickness of the bottom layer, $\bar{h}$, obtained using different mesh densities. The rest of the parameters are $R e=100, A t=0.2, R i=0.1, m=2, \kappa=0, B n=10, n=1.1$ and $\epsilon=10^{-9}$. The dotted line in panel (a) represents the analytical solution of the plug-flow displacement given by $M_{t} / M_{0}=1-t H / L$. 
Fig. 5: The effects of viscosity regularization parameter $(\epsilon)$ on the spatio-temporal evolution of the $\phi$ contours obtained using the simple model: (a) $\epsilon=10^{-6}$, (b) $\epsilon=10^{-9}$ and (c) $\epsilon=10^{-12}$. The rest of the parameter values are $R e=100, R i=1, A t=0.2, m=2, \kappa=0.0075$, $B n=30$ and $n=1.1$.

Fig. 6: Spatio-temporal evolution of $\phi$ contours obtained using the (a) simple, (b) Bercovier and Engleman's, and (c) Papanastasiou's model. The rest of the parameter values are $R e=100, R i=0.5, A t=0.2, m=2$, $\kappa=0.0075, B n=30$ and $n=1.1$.

Fig. 7: Spatio-temporal evolution of the unyielded domains obtained, shown in black, using (a) simple model, (b) Bercovier and Engleman's model and (c) Papanastasiou's model. The rest of the parameter values are the same as those used to generate Fig. 6 . The insets at the bottom represent the corresponding enlarged view of the region shown by rectangles.

Fig. 8: Contours of the index function, $\phi$ for (a) $B n=0$, (b) $B n=20$, (c) $B n=50$ and (d) $B n=100$ at $t=20$ and $t=30$. The rest of the parameters are $R e=100, A t=0.2, R i=1, \kappa=0.0075, m=2$ and $n=1$. Fig. 9: Unyielded domains, shown in black, for $B n=50$ at $t=20$ and $t=30$. The rest of the parameters are values are the same as those used in Fig. 8. The region marked by the rectangular box is shown as the inset in the third panel.

Fig. 10: Contours of the axial velocity, $u$, with position of the interface (shown by black solid line) for (a) $B n=0$, (b) $B n=20$, (c) $B n=50$ and 
(d) $B n=100$ at $t=20$ and $t=30$. The rest of the parameter values are the same as those used in Fig. 8.

Fig. 11: (a) Temporal variation of volume fraction of the displaced fluid $\left(M_{t} / M_{0}\right)$, (b) the rate of displacement, $\left(M_{t} / M_{0}\right)^{\prime}$, and (c) the average residual thickness of the bottom layer, $\bar{h}$, for different values of $B n$. Here (') represents the the derivative with respect to time. The rest of the parameters values are the same as those used in Fig. 8.

Fig. 12: Contours of the index function, $\phi$ (top), and unyielded surface (bottom) for (a) $n=0.7$, (b) $n=1$ and (c) $n=1.3$ at $t=20$ and $t=30$.

The rest of the parameters are $R e=100, A t=0.2, R i=1, \kappa=0.0075$, $m=2$ and $B n=30$.

Fig. 13: Contours of the axial velocity, $u$, with position of the interface (shown by black solid line) for (a) $n=0.7$, (b) $n=1$ and (c) $n=1.3$ at $t=20$ and $t=30$. The rest of the parameter values are the same as those used in Fig. 12.

Fig. 14: (a) The rate of displacement, $\left(M_{t} / M_{0}\right)^{\prime}$, and (b) the average residual thickness of the bottom layer, $\bar{h}$, for different values of $n$. Here $\left({ }^{\prime}\right)$ represents the the derivative with respect to time. The rest of the parameters values are the same as those used in Fig. 12. 
Figure 1

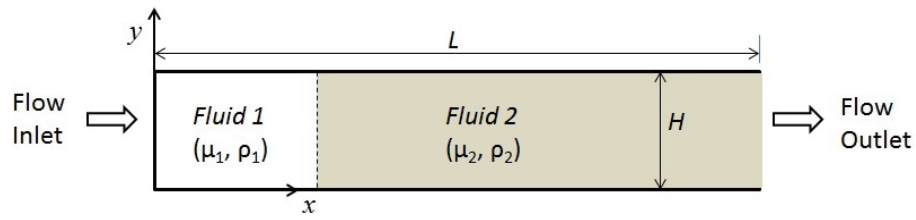


Figure 2

(a)

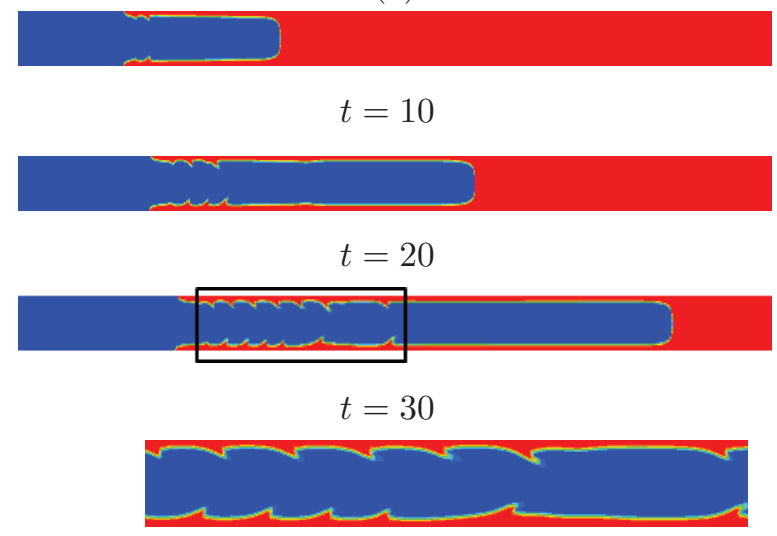

(b)

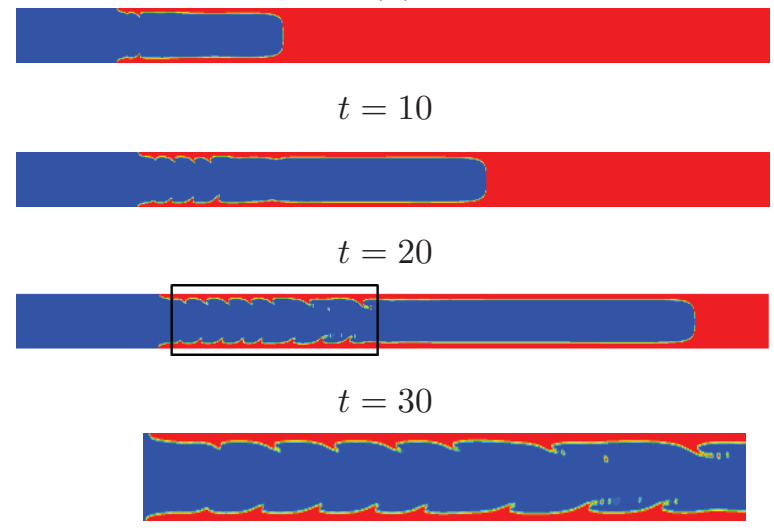

(c)

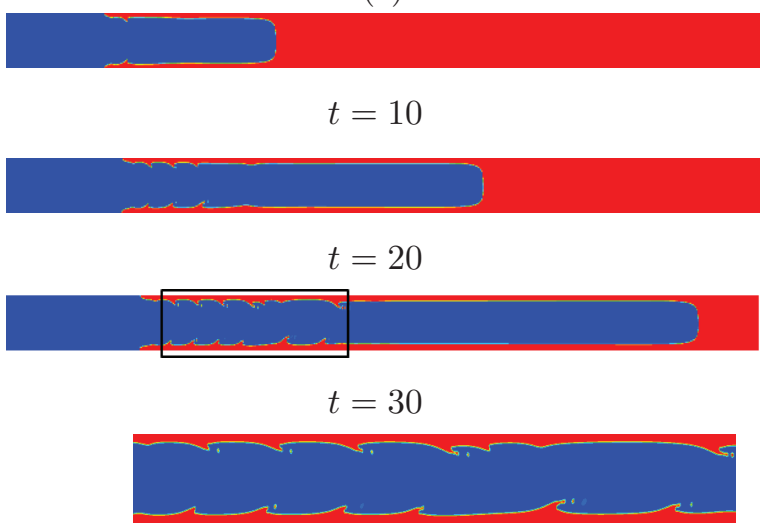


Figure 3

(a)

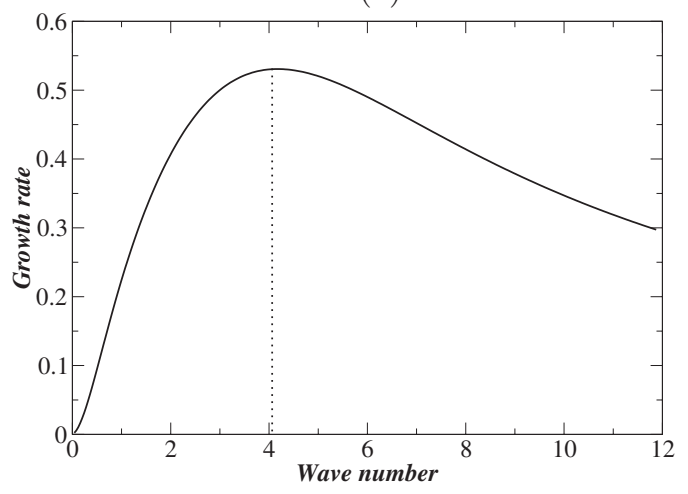

(b)

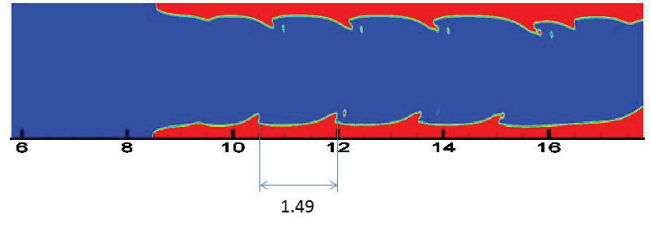


Figure 4

(a)

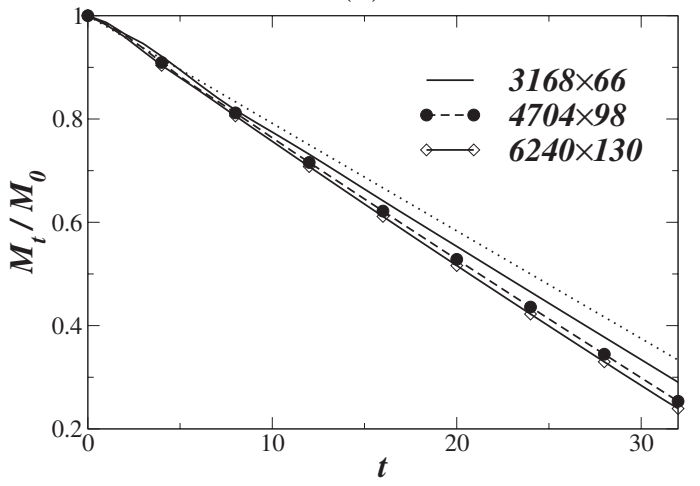

(b)

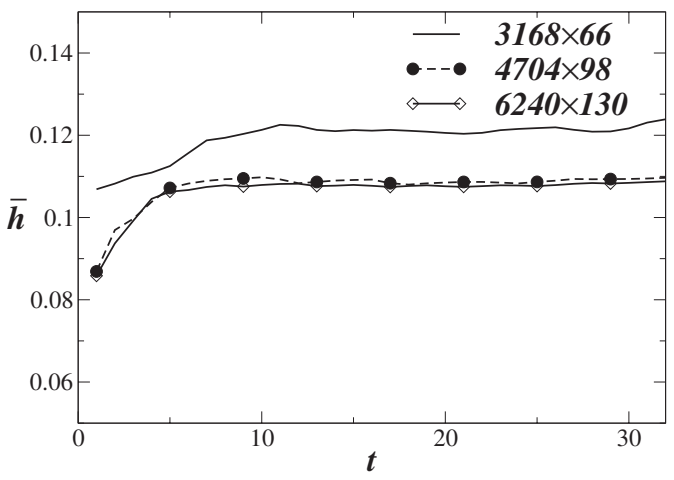


Figure 5

(a)

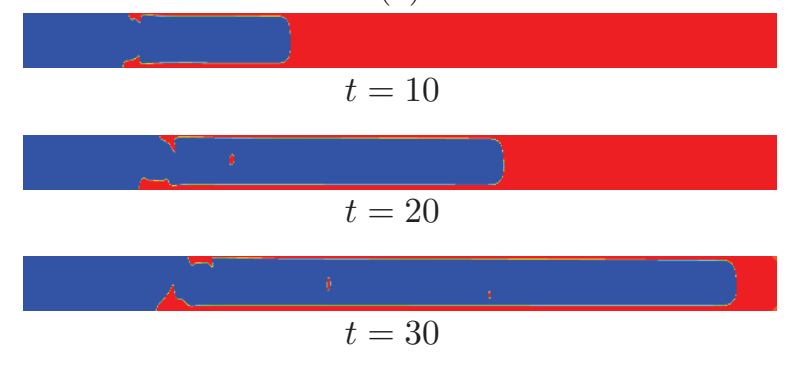

(b)

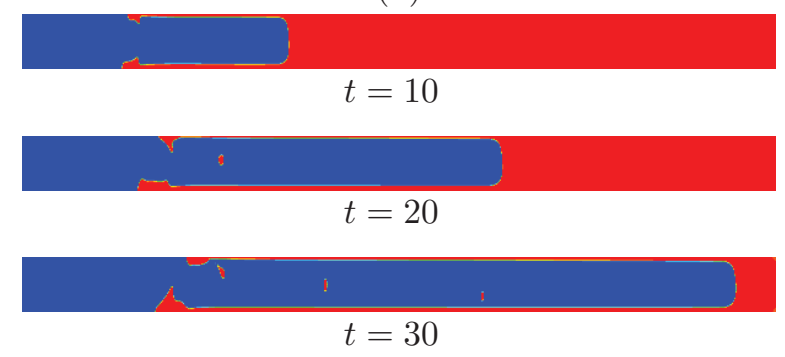

(c)

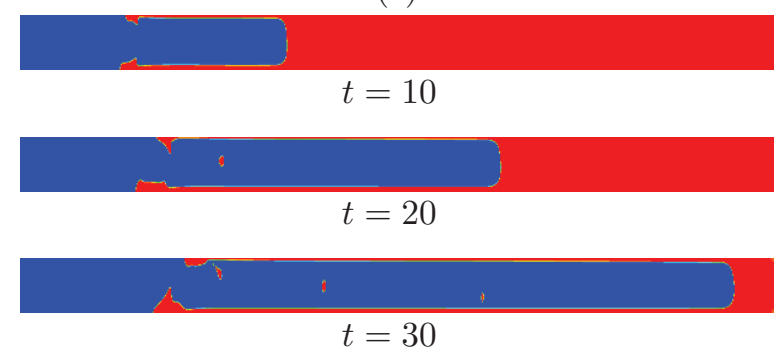


Figure 6

(a)

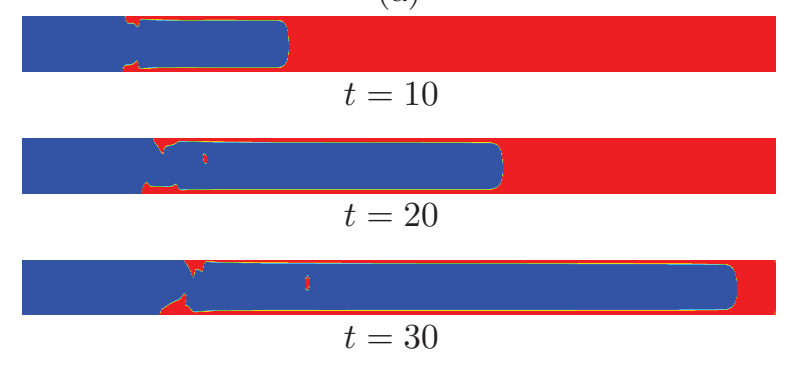

(b)

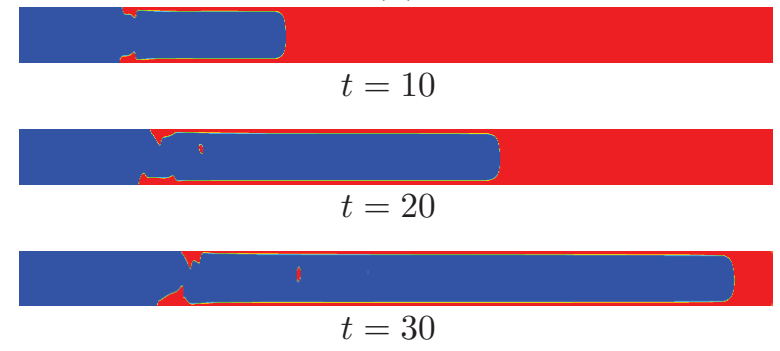

(c)

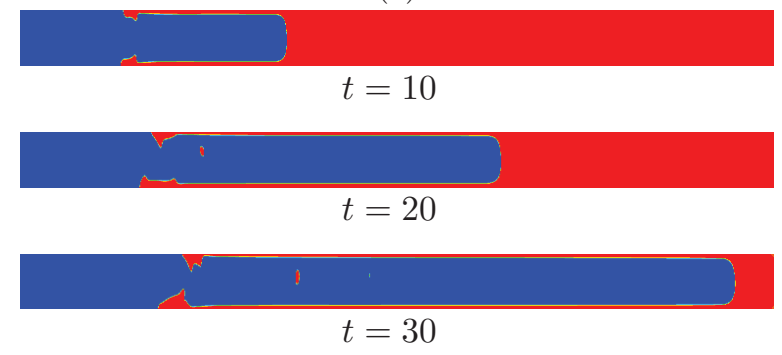


Figure 7

(a)

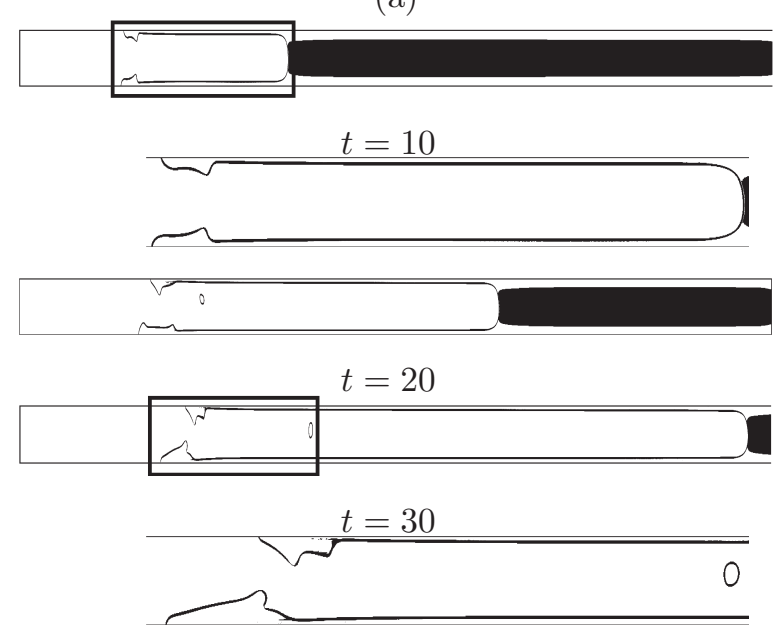

(b)

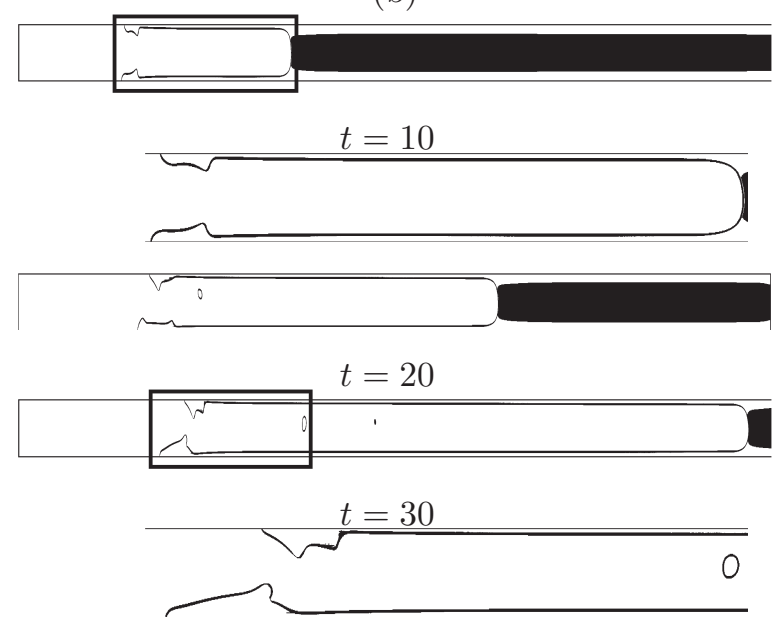

(c)

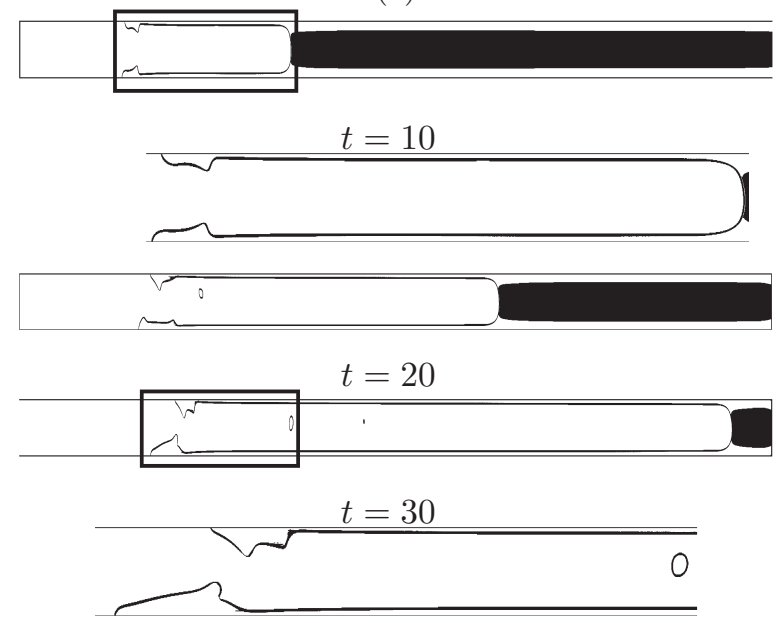


Figure 8

(a)

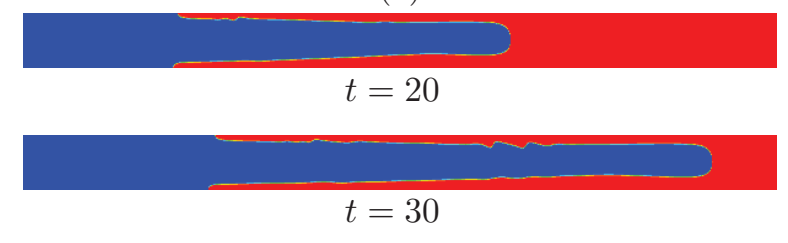

(b)

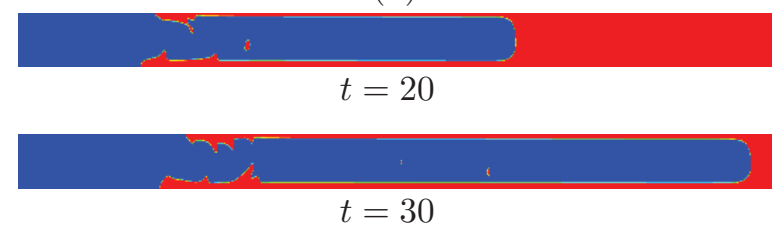

(c)

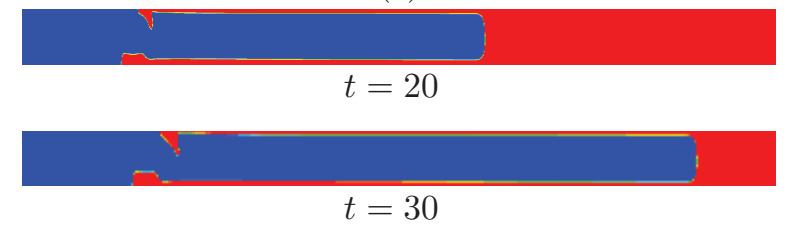

(d)

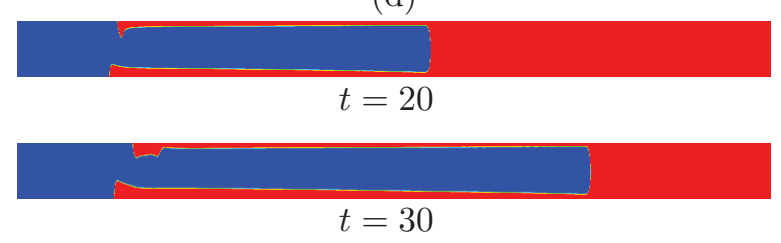

$t=30$ 
Figure 9

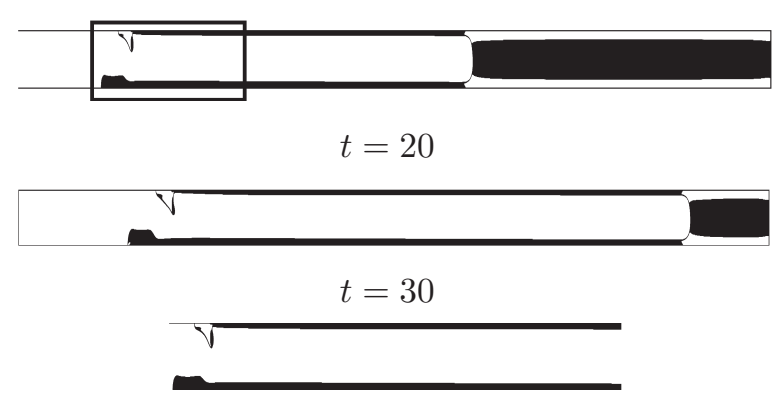


Figure 10

(a)

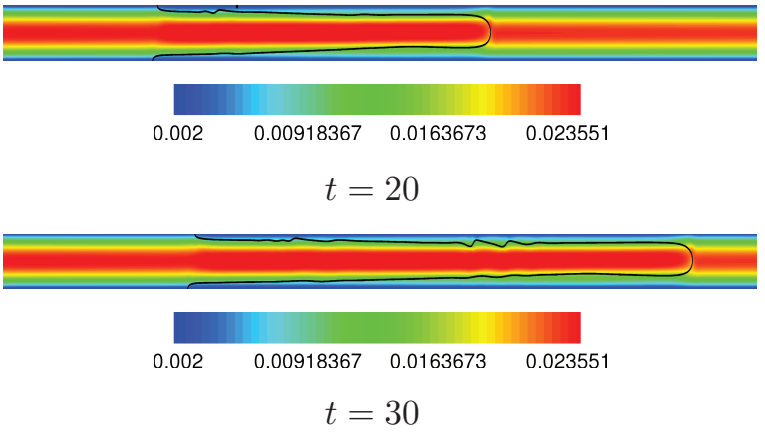

(b)

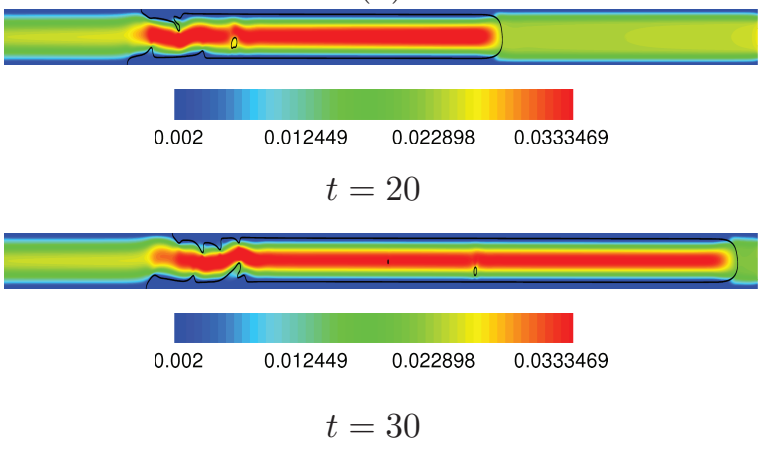

(c)
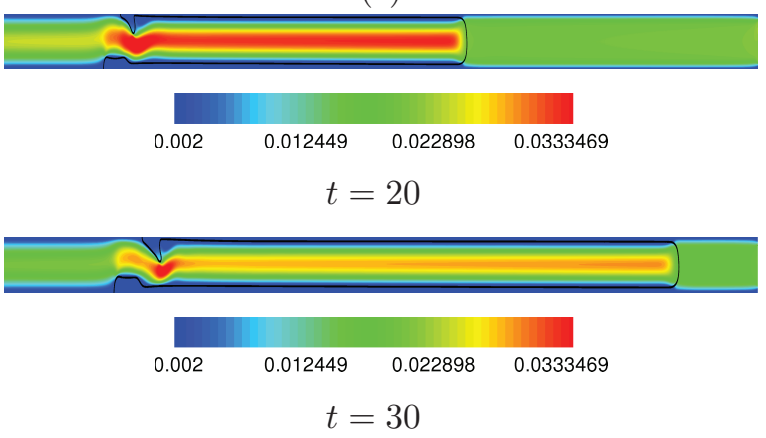

(d)

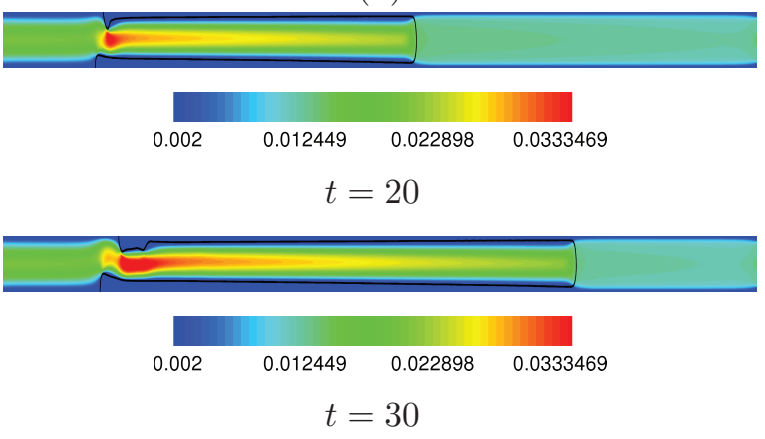


Figure 11

(a)

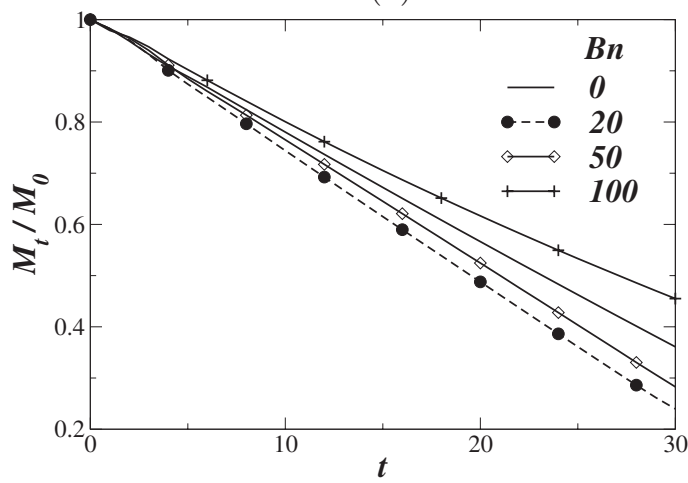

(b)

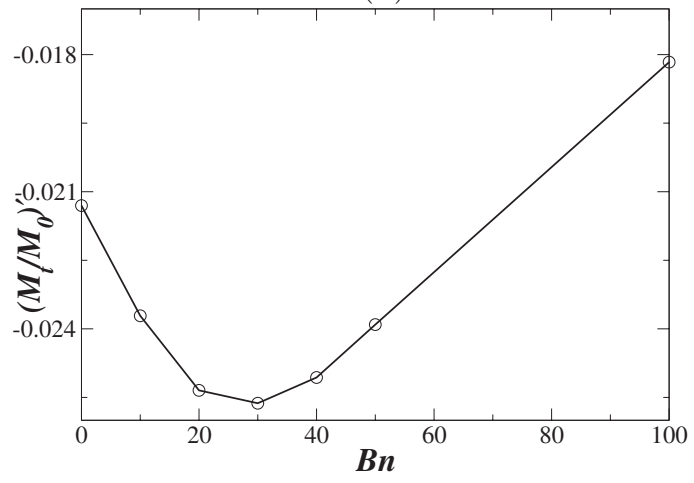

(c)

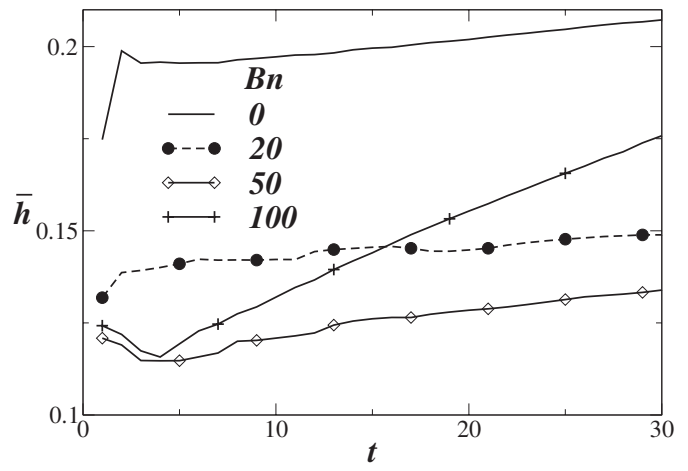


(a)

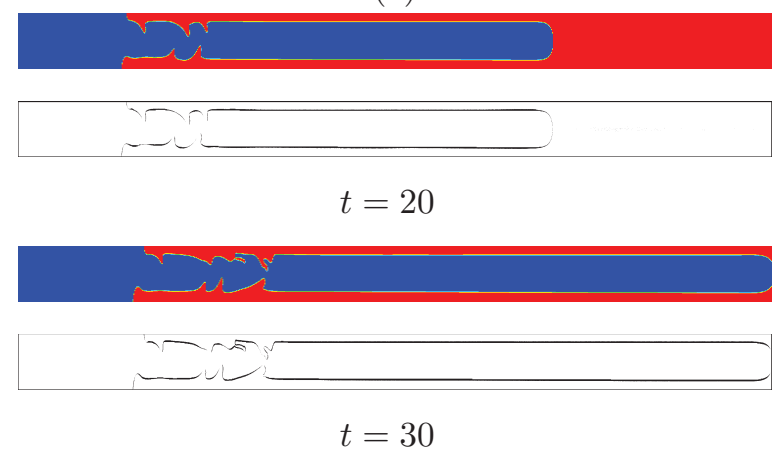

(b)

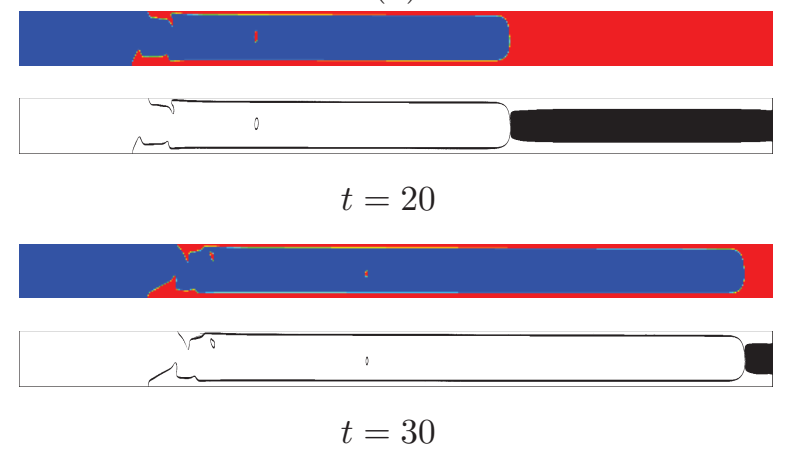

(c)
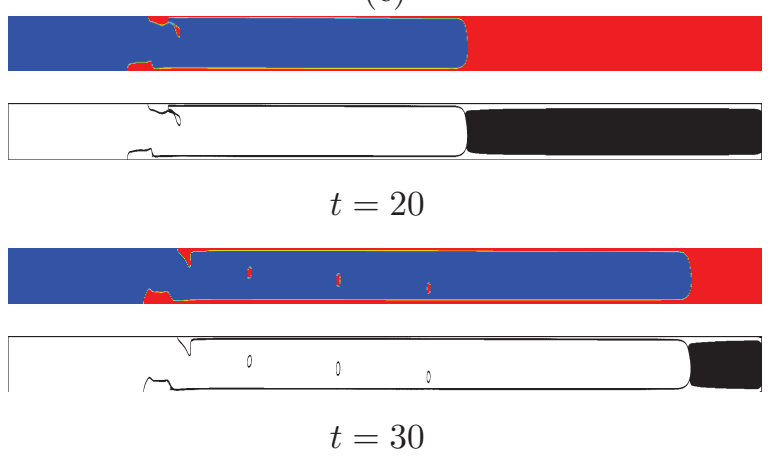
Figure 13

(a)

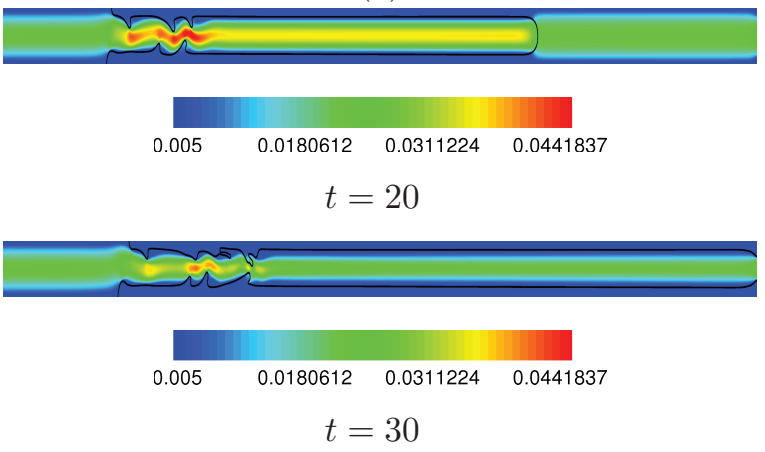

(b)

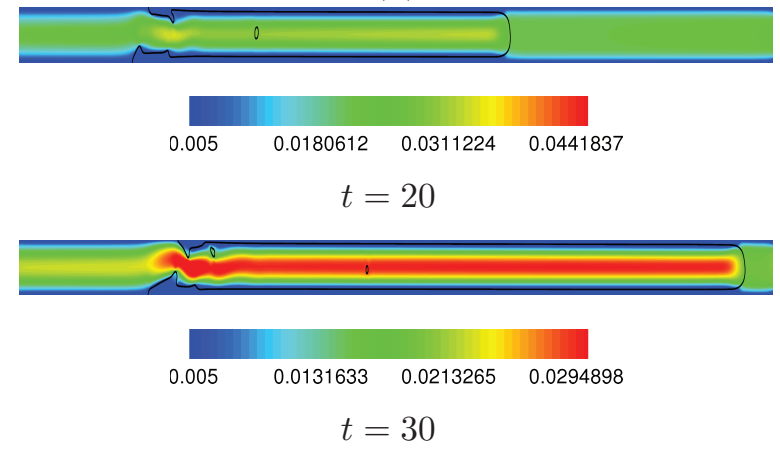

(c)

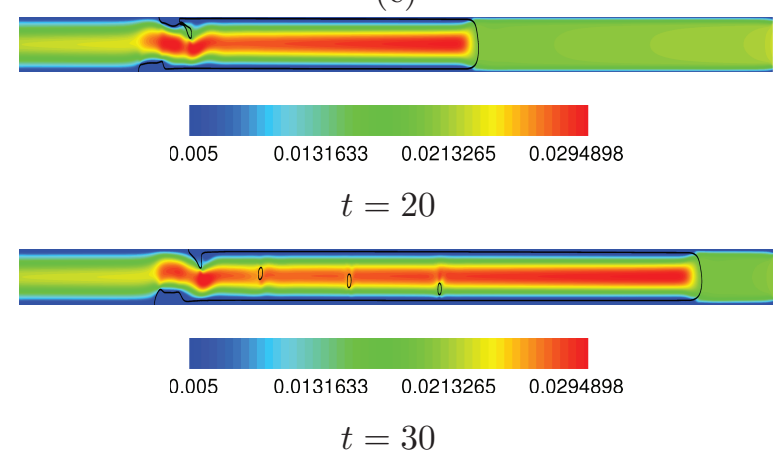


Figure 14

(a)

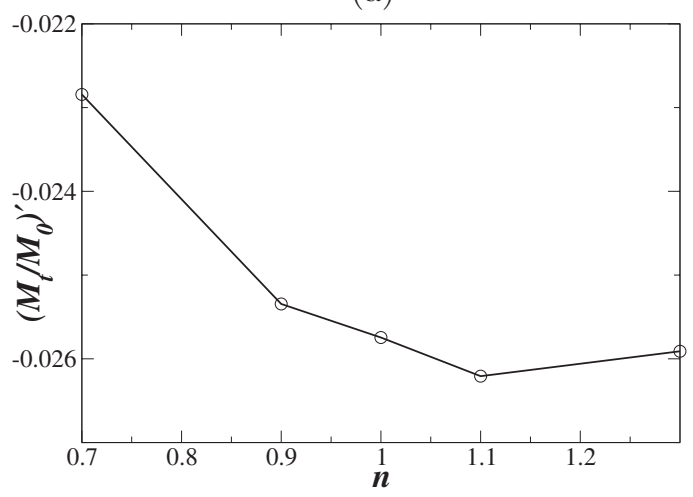

(b)

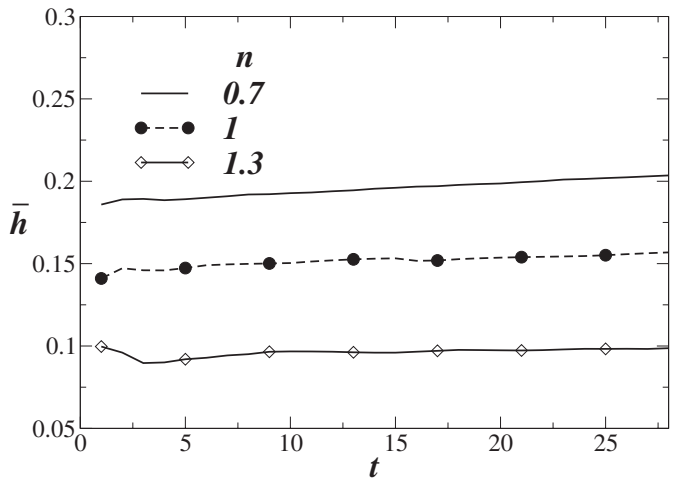

